\title{
Rates of Convergence of Gauss, Lobatto, and Radau Integration Rules for Singular Integrands
}

\author{
By Philip Rabinowitz
}

\begin{abstract}
Rates of convergence (or divergence) are obtained in the application of Gauss, Lobatto, and Radau integration rules to functions with an algebraic or logarithmic singularity inside, or at an endpoint of, the interval of integration. A typical result is the following: For a generalized Jacobi weight function on $[-1,1]$, the error in applying an $n$-point rule to $f(x)=|x-y|^{-\delta}$ is $O\left(n^{-2+2 \delta}\right)$, if $y= \pm 1$ and $O\left(n^{-1+\delta}\right)$ if $y \in(-1,1)$, provided we avoid the singularity. If we ignore the singularity $y$, the error is $O\left(n^{-1+2 \delta}(\log n)^{\delta}(\log \log n)^{\delta(1+\varepsilon)}\right)$ for almost all choices of $y$. These assertions are sharp with respect to order.
\end{abstract}

1. Introduction. This paper is a sequel to that of Lubinsky and myself [2] on the rates of convergence of Gauss integration rules for singular integrands. In this paper we extend the results in [2] in several directions. On the one hand, we extend the results for Gauss integration rules to results for Lobatto and Radau rules. This extension follows easily from the generalized Markov-Stieltjes inequality for Lobatto and Radau rules given in Lemma 3.2 and from a representation of the coefficients (or weights or Cotes numbers) in the Lobatto and Radau rules in terms of the coefficients in the Gauss rule with respect to a related weight function. A second extension is to the special case of Gauss-Jacobi integration rules as well as LobattoJacobi and Radau-Jacobi rules for certain values of the parameters defining the Jacobi weight function

$$
\omega^{(\alpha, \beta)}(x)=(1-x)^{\alpha}(1+x)^{\beta}, \quad \alpha, \beta>-1 .
$$

In [2], there were some results for $\alpha, \beta= \pm \frac{1}{2}$. In this paper, there are similar results for

$$
-1<\alpha=\beta \text { and }-\frac{1}{2} \leqslant \alpha, \beta \leqslant \frac{1}{2}
$$

in the Gauss case, and for other ranges of $\alpha$ and $\beta$ in the Lobatto and Radau cases. These results are based on the results in [5], where convergence and divergence theorems were proved for Gauss-Jacobi rules but no stress was placed on the rates of convergence as in this paper and in [2]. Finally, in dealing with endpoint singularities, we generalize the weight functions considered in [2] to the generalized Jacobi weight functions studied by Nevai and others [3].

Since this paper follows closely on [2], we shall only state the theorems generalizing those given therein but shall not, in general, give proofs, since they are almost identical word-for-word with those in [2], the major difference being that we shall

Received September 3, 1985.

1980 Mathematics Subject Classification. Primary 65D32. 
use $\omega(x) d x$ in place of $d \alpha(x)$ used in [2]. Of course, where there are some differences in the proofs, we shall indicate this. In Section 2, we shall establish our notation and introduce the integration rules. In Section 3, we shall prove the generalized Markov-Stieltjes inequality for Lobatto and Radau rules (Lemmas 3.2 and 3.3), and an additional basic lemma. In Sections 4 and 5, we study rates of convergence of our rules for functions with an interior singularity which satisfy certain monotonicity conditions, in particular, the functions $|x-y|^{-\delta}, 0<\delta<1$, and $-\log |x-y|$. In Section 6, we do the same for functions with endpoint singularities. Finally, in Sections 7 and 8, we generalize the results of Sections 4-6 to more general functions. This is the same structure as in [2], and will facilitate reference to that paper.

2. Notation. Let $[a, b]$ be a finite interval and $r, s \in\{0,1\}$. We shall be concerned with the approximation of the integral

$$
I[f]=\int_{a}^{b} f(x) \omega(x) d x
$$

by numerical integration rules of the form

$$
I_{n}[f]=\sum_{i=1-r}^{n+s} \lambda_{n i} f\left(x_{n i}\right) .
$$

Here, $\omega(x)$ is a nonnegative weight function which is positive over a subinterval of $[a, b]$ (usually almost everywhere) and in $L_{1}(a, b)$, and is such that $I[f]$ is properly or improperly Riemann-integrable. The points $x_{n i}$ are the zeros of

$$
(1+x)^{r}(1-x)^{s} p_{n}(x)
$$

where $p_{n}(x)=k_{n} x^{n}+\cdots, k_{n}>0$, belongs to the family of orthonormal polynomials with respect to the weight function

$$
\bar{\omega}(x)=(1+x)^{r}(1-x)^{s} \omega(x),
$$

and are ordered as follows:

$$
a=x_{n 0}<x_{n 1}<\cdots<x_{n n}<x_{n, n+1}=b .
$$

The coefficients $\lambda_{n i}$ are interpolatory and are given explicitly in terms of the $p_{n}(x)$ by

$$
\lambda_{n i}=\bar{\lambda}_{n i}\left(1+x_{n i}\right)^{-r}\left(1-x_{n i}\right)^{-s}, \quad i=1, \ldots, n,
$$

where the $\bar{\lambda}_{n i}$ are the Christoffel numbers

$$
\bar{\lambda}_{n i}=\left(\sum_{k=0}^{n-1} p_{k}^{2}\left(x_{n i}\right)\right)^{-1}, \quad i=1, \ldots, n,
$$

while if $r=1,(b-a)^{s} \lambda_{n 0}=I\left((b-x)^{s}\right)-\sum_{j=1}^{n} \lambda_{n j}\left(b-x_{n j}\right)^{s}$, and if $s=1$, $(b-a)^{r} \lambda_{n, n+1}=I\left((x-a)^{r}\right)-\sum_{j=1}^{n} \lambda_{n j}\left(x_{n j}-a\right)^{r}$.

For $r=s=0,(2.3)$ reduces to the Gauss rule; for $r=1, s=0$, to the left Radau rule; for $r=0, s=1$, to the right Radau rule; for $r=s=1$, to the Lobatto rule. If we define

$$
E_{n}[f]=I[f]-I_{n}[f],
$$

then $E_{n}[f]=0$ whenever $f$ is a polynomial of degree less than $2 n+r+s$. 
We shall frequently need to consider some fixed point $y \in(a, b)$ at which $f(x)$ may, or may not, have a singularity. Throughout $x_{c(n)}, x_{l(n)}, x_{r(n)}$ denote the points from $\left\{x_{n 0}, x_{n 1}, \ldots, x_{n n}, x_{n, n+1}\right\}$ which are, respectively, the closest to $y$, the closest from the left to $y$, and the closest from the right to $y$. More precisely,

$$
\begin{aligned}
\left|x_{c(n)}-y\right| & =\min \left\{\left|x_{n j}-y\right|: j=0,1, \ldots, n+1\right\}, \\
y-x_{l(n)} & =\min \left\{y-x_{n j}: x_{n j} \leqslant y\right\}, \\
x_{r(n)}-y & =\min \left\{x_{n j}-y: x_{n j}>y\right\} .
\end{aligned}
$$

When $x_{c(n)}$ is not uniquely defined by the above, which is the case only when $y$ is midway between $x_{l(n)}$ and $x_{r(n)}$, we take $x_{c(n)}=x_{l(n)}$. We let

$$
I_{n}^{*}[f]=\sum_{\substack{j=1 \\ j \neq c(n)}}^{n} \lambda_{n j} f\left(x_{n j}\right),
$$

so that $I_{n}^{*}$ avoids the singularity by omitting the closest abscissa to it. Further, we let

$$
E_{n}^{*}[f]=I[f]-I_{n}^{*}[f]
$$

Similarly, we define

$$
I_{n}^{* *}[f]=\sum_{\substack{j=1 \\ j \neq l(n), r(n)}}^{n} \lambda_{n j} f\left(x_{n j}\right),
$$

so that $I_{n}^{* *}$ avoids the singularity by omitting the closest abscissas from the left and right to $y$. Further,

$$
E_{n}^{* *}[f]=I[f]-I_{n}^{* *}[f]
$$

We let $\lambda_{c(n)}, \lambda_{l(n)}, \lambda_{r(n)}$ denote the coefficients corresponding to $x_{c(n)}, x_{l(n)}, x_{r(n)}$, respectively. Similarly, $x_{c(n) \pm 1}, \lambda_{c(n) \pm 1}$ denote $x_{n, c(n) \pm 1}$ and $\lambda_{n, c(n) \pm 1}$ and so on. Note that $x_{r(n)}=x_{l(n)+1}$.

Definition 2.1. We shall say that the integral of $\omega(x)$ is bounded above and below near $y, \omega \in I B(y)$, if there exist positive constants $m$ and $M$ such that

$$
m \leqslant\left(x_{2}-x_{1}\right)^{-1} \int_{x_{1}}^{x_{2}} \omega(x) d x \leqslant M
$$

for all $x_{1}, x_{2}$ in a neighborhood of $y$.

Thus the Jacobi weight $\omega^{(\alpha, \beta)}(x) \in I B(y)$ for all $y \in(-1,1)$.

The usual symbols $O, o, \sim, \cong$ will be used to compare sequences and functions. For example, if $\left(c_{n}\right),\left(d_{n}\right)$ are sequences of real numbers,

$$
\begin{aligned}
& c_{n}=O\left(d_{n}\right) \Leftrightarrow \limsup _{n \rightarrow \infty}\left|c_{n} / d_{n}\right|<\infty, \\
& c_{n}=o\left(d_{n}\right) \Leftrightarrow \lim _{n \rightarrow \infty} c_{n} / d_{n}=0, \\
& c_{n} \cong d_{n} \Leftrightarrow \lim _{n \rightarrow \infty} c_{n} / d_{n}=1, \\
& c_{n} \sim d_{n} \Leftrightarrow K_{1} \leqslant c_{n} / d_{n} \leqslant K_{2},
\end{aligned}
$$

for all large enough $n$, where $K_{1}$ and $K_{2}$ are positive constants. 
Definition 2.2. Let $\mathscr{G}$ be a finite real closed interval. If $f \in C[\mathscr{G}]$, the modulus of continuity of $f$ in $\mathscr{G}$ is

$$
\omega_{f}(\mathscr{G} ; \varepsilon)=\max \left\{\left|f\left(x_{1}\right)-f\left(x_{2}\right)\right|:\left|x_{1}-x_{2}\right| \leqslant \varepsilon ; x_{1}, x_{2} \in \mathscr{G}\right\} \quad \text { for any } \varepsilon>0 .
$$

We say $f \in \operatorname{Lip}(\theta)$ in $\mathscr{G}$ where $0<\theta \leqslant 1$ if $\omega_{f}(\mathscr{G} ; \varepsilon)=O\left(\varepsilon^{\theta}\right)$, and we say $f \in \operatorname{Lip}(\theta ; \eta)$ in $\mathscr{G}$ where $\theta \geqslant 0$ and $\eta$ is real if $\omega_{f}(\mathscr{G} ; \varepsilon)=O\left(\varepsilon^{\theta}|\log \varepsilon|^{-\eta}\right)$.

Definition 2.3. Let $\mathscr{G}$ be a real interval. Let $k$ be a positive integer. We shall say $f$ : $\mathscr{G} \rightarrow \mathbf{R}$ is $k$-absolutely monotone in $\mathscr{G}(k$-completely monotone in $\mathscr{G})$ if

$$
\begin{aligned}
& f^{(j)}(x) \geqslant 0, \quad x \in \mathscr{G}, j=0,1,2, \ldots, k, \\
& \left((-1)^{j} f^{(j)}(x) \geqslant 0, x \in \mathscr{G}, j=0,1,2, \ldots, k\right) .
\end{aligned}
$$

If $f$ is $k$-absolutely monotone in $\mathscr{G}(k$-completely monotone in $\mathscr{G})$ for all positive integers $k$, we shall say $f$ is absolutely monotone in $\mathscr{G}$ (completely monotone in $\mathscr{G}$ ).

3. Basic Lemmas. The Markov-Stieltjes inequalities in Lemmas 3.2 and 3.3 depend on the following fundamental lemma:

LEMMA 3.1. Let $f$ be $(m+1)$-absolutely monotone in $[a, \xi]$ with strict inequality holding in (2.7). Let $P(x)$ be a polynomial of degree at most $m$. Let

$$
\begin{aligned}
& m_{1}=\text { total multiplicity of zeros of } f-P \text { in }[a, \xi], \\
& m_{2}=\text { total multiplicity of zeros of } P \text { in }[\xi, b] .
\end{aligned}
$$

Then $m_{1}+m_{2} \leqslant m+1$.

Proof. Freud [1, Lemma I.5.3] gives a proof for the case $(-\infty, \xi]$ and $[\xi, \infty)$. By substituting $[a, \xi]$ and $[\xi, b]$ for $(-\infty, \xi]$ and $[\xi, \infty)$ respectively throughout his proof, we see that our statement is also true.

LEMMA 3.2. Let $f(x)$ be $(2 n-1+s)$-absolutely monotone in $\left[a, x_{n k}\right)$ for some $n \geqslant 1,1-r \leqslant k \leqslant n+s$. Then

(i)

$$
\sum_{j=1-r}^{k-1} \lambda_{n j} f\left(x_{n j}\right) \leqslant \int_{a}^{x_{n k}} f(x) \omega(x) d x .
$$

(ii) If , in addition, $f(x)$ is $(2 n-1+s)$-absolutely monotone in $\left[a, x_{n k}\right]$, then

$$
\sum_{j=1-r}^{k} \lambda_{n j} f\left(x_{n j}\right) \geqslant \int_{a}^{x_{n k}} f(x) \omega(x) d x .
$$

In particular,

$$
\sum_{j=1-r}^{k-1} \lambda_{n j} \leqslant \int_{a}^{x_{n k}} \omega(x) d x \leqslant \sum_{j=1-r}^{k} \lambda_{n j}, \quad 1 \leqslant k \leqslant n+s .
$$

Proof. (i) Define a polynomial $p(x)$ of degree $\leqslant 2 n-2+s$ by the $2 n-1+s$ interpolation conditions

$$
\begin{aligned}
p\left(x_{n j}\right) & = \begin{cases}f\left(x_{n j}\right), & j=1,2, \ldots, k-1, \\
0, & j=k, k+1, \ldots, n+s ;\end{cases} \\
p^{\prime}\left(x_{n j}\right) & = \begin{cases}f^{\prime}\left(x_{n j}\right), & j=1,2, \ldots, k-1, \\
0, & j=k+1, k+2, \ldots, n .\end{cases}
\end{aligned}
$$


We shall assume initially that strict inequality holds in (2.7). Let $\xi \in\left(x_{n, k-1}, x_{n k}\right)$. Then by $(3.1 \mathrm{~A}, \mathrm{~B}), f-p$ has $m_{1} \geqslant 2 k-2$ zeros in $[a, \xi]$ and $p$ has $m_{2} \geqslant 2 n-2 k$ $+1+s$ zeros in $[\xi, b]$. Thus $m_{1}+m_{2} \geqslant 2 n-1+s=\operatorname{deg}(p)+1$. By Lemma 3.1, we have $m_{1}+m_{2} \leqslant 2 n-1+s$. Thus, $m_{1}=2 k-2$ and $m_{2}=2 n-2 k+1+s$, and the only zeros of $f-p$ and $p$ in $[a, \xi]$ and $[\xi, b]$, respectively, are already listed in (3.1A, B). As all zeros of $f-p$ in $(a, \xi]$ are double zeros, it follows that $f-p$ does not change sign in $(a, \xi]$ for any $\xi<x_{n k}$, and hence $f-p$ does not change sign in $\left(a, x_{n k}\right)$. As $p\left(x_{n k}\right)=0$, we deduce

$$
f(x) \geqslant p(x), \quad x \in\left[a, x_{n k}\right) .
$$

Next, as $\xi>x_{n, k-1}$ was arbitrary, it follows $p(x)$ has $2 n-2 k+1+s$ zeros in $\left(x_{n, k-1}, b\right]$, these being listed in (3.1A, B). Since $p\left(x_{n, k-1}\right)=f\left(x_{n, k-1}\right)>0$ and as $p(x)$ has a simple zero at $x_{n k}$ and double zeros at $x_{n j}, j=k+1, k+2, \ldots, n$, it follows that $p(x)$ changes sign at $x_{n k}$ and

$$
0 \geqslant p(x), \quad x \in\left[x_{n k}, b\right] .
$$

Then by (3.2) and (3.3), and by (3.1A),

$$
\int_{a}^{x_{n k}} f(x) \omega(x) d x \geqslant \int_{a}^{b} p(x) \omega(x) d x=\sum_{j=1-r}^{n+s} \lambda_{n j} p\left(x_{n j}\right)=\sum_{j=1-r}^{k-1} \lambda_{n j} f\left(x_{n j}\right) .
$$

Finally, if strict inequality does not hold in (2.7), $f_{\varepsilon}(x)=f(x)+\varepsilon e^{x}$ satisfies (2.7) with strict inequality for any $\varepsilon>0$. Applying the above inequality to $f_{\varepsilon}$, and letting $\varepsilon \rightarrow 0+$, we obtain the more general inequality.

(ii) is similar: One defines a polynomial $P(x)$ of degree $\leqslant 2 n-2+s$ by

$$
\begin{aligned}
& P\left(x_{n j}\right)= \begin{cases}f\left(x_{n j}\right), & j=1,2, \ldots, k, \\
0, & j=k+1, k+2, \ldots, n+s ;\end{cases} \\
& P^{\prime}\left(x_{n j}\right)= \begin{cases}f^{\prime}\left(x_{n j}\right), & j=1,2, \ldots, k-1, \\
0, & j=k+1, k+2, \ldots, n,\end{cases}
\end{aligned}
$$

and uses Lemma 3.1 to deduce

$$
\begin{array}{ll}
f(x) \leqslant P(x), & x \in\left[a, x_{n k}\right], \\
0 \leqslant P(x), & x \in\left[x_{n k}, b\right] .
\end{array}
$$

For $(2 n-1+r)$-completely monotone functions, there is the following corollary.

LEMMA 3.3. Let $f(x)$ be $(2 n-1+r)$-completely monotone in $\left(x_{n k}, b\right]$ for some $n \geqslant 1,1 \leqslant k \leqslant n$. Then

(i)

$$
\sum_{j=k+1}^{n+s} \lambda_{n j} f\left(x_{n j}\right) \leqslant \int_{x_{n k}}^{b} f(x) \omega(x) d x .
$$

(ii) If, in addition, $f(x)$ is $(2 n-1+r)$-completely monotone in $\left[x_{n k}, b\right]$, then

$$
\sum_{j=k}^{n+s} \lambda_{n j} f\left(x_{n j}\right) \geqslant \int_{x_{n k}}^{b} f(x) \omega(x) d x \text {. }
$$

Proof. See [2].

The following lemma on the asymptotic behavior of coefficients and abscissas will be useful in the sequel. 
Lemma 3.4. Assume that $\omega(x) \in I B(y)$ for $y \in(a, b)$. Then there exist positive constants $c_{1}, c_{2}, c_{3}, c_{4}$ and a neighborhood $\mathscr{G}$ of $y$ such that for all $n$ and $j, 1 \leqslant j \leqslant n$,

(i) $x_{n j} \in \mathscr{G} \Rightarrow c_{1} / n \leqslant x_{n, j+1}-x_{n j} \leqslant c_{2} / n$,

(ii) $x_{n j} \in \mathscr{G} \Rightarrow c_{3} / n \leqslant \lambda_{n j} \leqslant c_{4} / n$.

Proof. (i) See [2].

(ii) By Lemma 3.4(ii) in [2], the Christoffel numbers $\bar{\lambda}_{n j}$ satisfy (ii). Hence, by (2.4), (ii) holds.

4. Interior Singularities, Part 1. In this section, we investigate the asymptotic behavior of $E_{n}[f]$, where $f(x)=|x-y|^{-\delta}$ or $-\log |x-y|$. First, however, we establish our basic error estimate which may be applied to functions with a singularity on either one or both sides of $y$.

LEMMA 4.1. Let $f(x)$ be $(2 n-1+s)$-absolutely monotone in $[a, y)$ and $(2 n-1$ $+r$ )-completely monotone in $(y, b]$. Then

(i)

$$
\int_{x_{/(n)}}^{x_{r(n)}} f(x) \omega(x) d x \leqslant E_{n}^{* *}[f] \leqslant \int_{x_{/(n)-1}}^{x_{r(n)+1}} f(x) \omega(x) d x .
$$

(ii) If $y \neq x_{c(n)}$,

$$
\int_{x_{/(n)}}^{x_{r(n)}} f(x) \omega(x) d x-\sum_{j=l(n)}^{r(n)} \lambda_{n j} f\left(x_{n j}\right) \leqslant E_{n}[f] \leqslant \int_{x_{/(n)}}^{x_{r(n)}} f(x) \omega(x) d x .
$$

(iii) If $j$ is the integer such that $j \in\{l(n), r(n)\} \backslash\{c(n)\}$, then

$$
E_{n}^{*}[f]=E_{n}^{* *}[f]-\lambda_{n j} f\left(x_{n j}\right) \text {. }
$$

(iv) If $y=x_{c(n)}$,

$$
0 \leqslant E_{n}^{*}[f] \leqslant \int_{x_{/(n)-1}}^{x_{r(n)}} f(x) \omega(x) d x
$$

\section{Proof. See [2].}

We can now prove a general theorem for "2-sided" singularities:

TheOREM 4.2. Let $(a, b)$ be a finite interval and $y \in(a, b)$. Let $\omega(x) \in I B(y)$. Let $f(x)$ be absolutely monotone in $[a, y)$, completely monotone in $(y, b]$, and let $f(y)=0$. Further assume $f(x)$ grows at roughly the same rate on both sides of $y$ as $x \rightarrow y$, that is

$$
f(y-u) \sim f(y+u) \quad \text { as } u \rightarrow 0+.
$$

Let $\mu_{n}=\int_{y-1 / n}^{v} f(x) d x, n=1,2,3, \ldots$ Then

(i) $E_{n}^{* *}[f] \sim \mu_{n}$,

(ii) $E_{n}^{*}[f]=O\left(\mu_{n}\right)$,

(iii) $E_{n}[f]=O\left(\mu_{n}\right)-\lambda_{c(n)} f\left(x_{c(n)}\right)$,

and $\lambda_{c(n)} \sim n^{-1}$.

Proof. See proof of Theorem 4.3 in [2].

Thus the rate of convergence to 0 of the error, where the singularity is avoided using $I_{n}^{*}$ or $I_{n}^{* *}$, is determined by the asymptotic behavior of $\mu_{n}$. As a first 
corollary, we have

COROllaRy 4.3. Let $(a, b)$ be a finite interval and $y \in(a, b)$. Let

$$
f(x)= \begin{cases}|x-y|^{-\delta}, & x \in(a, b) \backslash\{y\}, \\ 0, & x=y,\end{cases}
$$

where $0<\delta<1$. Assume $\omega \in I B(y)$. Then

(i) $E_{n}^{* *}[f] \sim n^{-1+\delta}$,

(ii) $E_{n}^{*}[f]=O\left(n^{-1+\delta}\right)$,

and there exists $\delta_{0} \in(0,1)$ such that, whenever $\delta \in\left(\delta_{0}, 1\right)$, we have

$$
E_{n}^{*}[f] \sim n^{-1+\delta} .
$$

(iii) For those positive integers $n$ for which $y \neq x_{c(n)}$,

$$
E_{n}[f]=-\lambda_{c(n)}\left|x_{c(n)}-y\right|^{-\delta}+O\left(n^{-1+\delta}\right)=O\left(n^{-1}\left|x_{c(n)}-y\right|^{-\delta}\right),
$$

where $\lambda_{c(n)} \sim n^{-1}$.

Proof. See proof of Corollary 4.4 in [2].

Next, we have a corollary for logarithmic singularities.

COROllary 4.4. Let $(a, b)$ be a finite interval and $y \in(a, b)$. Let

$$
f(x)= \begin{cases}-\log |x-y|, & x \in(a, b) \backslash\{y\}, \\ 0, & x=y .\end{cases}
$$

Assume $\omega \in I B(y)$. Then

(i) $E_{n}^{* *}[f] \sim n^{-1} \log n$.

(ii) $E_{n}^{*}[f]=O\left(n^{-1} \log n\right)$.

(iii) For those positive integers $n$ for which $y \neq x_{c(n)}$,

$$
E_{n}[f]=-\lambda_{c(n)} \log \left|x_{c(n)}-y\right|+O\left(n^{-1} \log n\right)=O\left(n^{-1} \log \left|x_{c(n)}-y\right|\right),
$$

where $\lambda_{c(n)} \sim n^{-1}$.

Proof. See proof of Corollary 4.5 in [2].

In our next corollary, we have the following analogue of Theorem 2 in Rabinowitz [4], for the case where $y=\cos (\pi p / q)$ with $p / q$ a rational number.

Corollary 4.5. Let $(a, b)=(-1,1)$ and let $\omega(x)$ be the Jacobi weight function $\omega^{(\alpha, \beta)}(x)$, where $\alpha+s, \beta+r= \pm 1 / 2$ and $\alpha, \beta>-1$. Let $y=\cos (\pi p / q)$, where $p / q$ is a rational number in $(0,1)$.

(i) If

$$
f(x)= \begin{cases}|x-y|^{-\delta}, & x \in(-1,1) \backslash\{y\}, \\ 0, & x=y,\end{cases}
$$

where $0<\delta<1$, then $E_{n}[f]=O\left(n^{-1+\delta}\right)$.

(ii) If

$$
f(x)= \begin{cases}-\log |x-y|, & x \in(-1,1) \backslash\{y\}, \\ 0, & x=y,\end{cases}
$$

then $E_{n}[f]=O\left(n^{-1} \log n\right)$. 
Proof. See proof of Corollary 4.6 in [2].

In our final corollary, we extend the results of Corollary 4.5 for more general Jacobi weights.

COROLlaRY 4.6. Let $(a, b)=(-1,1)$ and let $\omega(x)$ be the Jacobi weight function $\omega^{(\alpha, \beta)}(x)$, where $\alpha$ and $\beta$ satisfy the following conditions in addition to the conditions $\alpha, \beta>-1$ :

$$
-1<\alpha+s=\beta+r \text { or } \quad-\frac{1}{2} \leqslant \alpha+s, \beta+r \leqslant \frac{1}{2} .
$$

Let $y=\cos (\pi p / q)$, where $p / q$ is a rational number in $(0,1)$. Then the conclusions of Corollary 4.5 hold.

Proof. In [5], it was shown that $\left|y-x_{c(n)}\right| \geqslant c / n$ for large $n$ such that $y \neq x_{c(n)}$. The rest of the proof is the same as that of Corollary 4.6 in [2].

5. Interior Singularities, Part 2. We now prove results of a different character to those of Section 4. For example, we show that, for almost all choices of $y$,

$$
E_{n}\left[|x-y|^{-\delta}\right]=O\left(n^{-1+2 \delta}(\log n)^{\delta}(\log \log n)^{\varepsilon \delta}\right) \text {, }
$$

where $\varepsilon>1$, and that this result is substantially the best possible. This is the analogue of Theorem 3 in Rabinowitz [4].

Theorem 5.1. (i) Assume $\omega(x) \in I B(y)$ for each $y$ interior to the interval $(a, b)$. Then, given $\varepsilon>1$, there is a set $\mathscr{E}_{\varepsilon}$ in $(a, b)$ of linear Lebesgue measure zero with the following property:

$$
E_{n}\left[|x-y|^{-\delta}\right]=O\left(n^{-1+2 \delta}(\log n)^{\delta}(\log \log n)^{\varepsilon \delta}\right)
$$

for all $0<\delta<1$, whenever $y \notin \mathscr{E}_{\varepsilon}$.

Hence, if $\delta<\frac{1}{2}, E_{n}\left[|x-y|^{-\delta}\right] \rightarrow 0$ as $n \rightarrow \infty$ for almost all $y \in(a, b)$.

(ii) Assume $(a, b)=(-1,1)$ and that $\omega(x)$ is the Jacobi weight function $\omega^{(\alpha, \beta)}(x)$, where $\alpha+s, \beta+r= \pm \frac{1}{2}$ and $\alpha, \beta>-1$. Then there is a set $\mathscr{E}$ in $(-1,1)$ of linear Lebesgue measure zero with the following property:

$$
E_{n}\left[|x-y|^{-\delta}\right] \geqslant c n^{-1+2 \delta}(\log n)^{\delta}(\log \log n)^{\delta}
$$

for infinitely many integers $n$ and for all $0<\delta<1$, whenever $y \notin \mathscr{E}$. Here $c$ is a positive constant independent of $n, y$ and $\delta$.

Hence, if $\delta \geqslant \frac{1}{2}, E_{n}\left[|x-y|^{-\delta}\right] \nrightarrow 0$ as $n \rightarrow \infty$ for almost all $y \in(-1,1)$.

(iii) Assume $(a, b)=(-1,1)$, and that $\omega(x)$ is the Jacobi weight function $\omega^{(\alpha, \beta)}(x)$, where $\alpha$ and $\beta$ satisfy (4.8). Then, if $y=\cos \pi \xi$, where $\xi$ is an irrational number, then

$$
\left|E_{n}\left[|x-y|^{-\delta}\right]\right| \geqslant c n^{-1+2 \delta}
$$

for infinitely many integers and all $0<\delta<1$.

Proof. (i) See [2].

(ii) See [2] with $\nu, \beta$ replaced by $\alpha+s, \beta+r$, respectively.

(iii) In [5], it was shown that $\left|y-x_{c(n)}\right| \leqslant c_{1} / n^{2}$ for infinitely many integers $n$. Applying Corollary 4.3(iii) and Lemma 3.4(ii), we have that

$$
\left|E_{n}\left[|x-y|^{-\delta}\right]\right|=\lambda_{c(n)}\left|x_{c(n)}-y\right|^{-\delta}+O\left(n^{-1+\delta}\right) \geqslant \frac{c_{3}}{n}\left(\frac{c_{1}}{n^{2}}\right)^{-\delta}=c n^{-1+2 \delta}
$$

for infinitely many $n$ and all $0<\delta<1$. 
Note that $(\log \log n)^{\varepsilon \delta}$ in (5.1) may be replaced by $(\log \log n)^{\delta}(\log \log \log n)^{\varepsilon \delta}$, and so on. Similar remarks apply to part (ii) of the above theorem. The proof of the following result is similar to that of Theorem 5.1.

TheOREM 5.2. Assume $\omega \in I B(y)$ for each $y$ interior to the finite interval $(a, b)$. Then there is a set $\mathscr{E}$ of linear Lebesgue measure zero (even further of Hausdorff dimension zero) such that $E_{n}[-\log |x-y|]=O\left(n^{-1} \log n\right)$ whenever $y \notin \mathscr{E}$.

The results in Theorems 5.1(i) and 5.2 are the best possible in that they cannot be improved to include all real numbers in $(a, b)$. This is shown by Example 1 in [5], where a number $\hat{y}$ and a subsequence $I_{n_{k}}[f]$ of the sequence of Gauss rules $(r=s=0)$ are constructed for which $I_{n_{k}}[\log |x-\hat{y}|]$ does not converge to $I[\log |x-\hat{y}|]$.

6. Endpoint Singularities. We discuss here the case where the singularity is at the right endpoint $b$. All the results can be easily rewritten for the case when the singularity is at $a$. If we define the value of the integrand at $b$ to be $f(b)=0$, we see that in all cases,

$$
I_{n}[f]=\sum_{i=1-r}^{n} \lambda_{n i} f\left(x_{n i}\right) .
$$

Nevertheless, in our next lemma, we shall have to distinguish between the cases $s=0$ and $s=1$. Even though the end result is the same, the proofs are different. Before stating our lemma, we note that for endpoint singularities, there is no need to omit any abscissas, so that we can restrict ourselves to the study of $E_{n}[f]$.

LEMMA 6.1. (a) Let $f(x)$ be $(2 n+r+s)$-absolutely monotone in $[a, b)$ such that $\lim _{x \rightarrow b} f(x)=\infty$ and define $f(b)=0$. Then

$$
\max \left\{\int_{x_{n n}}^{b} f(x) \omega(x) d x-\lambda_{n n} f\left(x_{n n}\right), 0\right\} \leqslant E_{n}[f] \leqslant \int_{x_{n n}}^{b} f(x) \omega(x) d x .
$$

(b) Let $f(x)$ be $(2 n+r+s)$-completely monotone in $(a, b]$ such that $\lim _{x \rightarrow a} f(x)$ $=\infty$ and define $f(a)=0$. Then

$$
\max \left\{\int_{a}^{x_{n 1}} f(x) \omega(x) d x-\lambda_{n 1} f\left(x_{n 1}\right), 0\right\} \leqslant E_{n}[f] \leqslant \int_{a}^{x_{n 1}} f(x) \omega(x) d x .
$$

Proof. (a) By Lemmas 3.2(i) and (ii),

$$
\begin{aligned}
\sum_{j=1}^{n-1} \lambda_{n j} f\left(x_{n j}\right) & \leqslant \int_{a}^{x_{n n}} f(x) \omega(x) d x \leqslant \sum_{j=1}^{n} \lambda_{n j} f\left(x_{n j}\right) \\
& \Rightarrow I_{n}[f]-\lambda_{n n} f\left(x_{n n}\right) \leqslant I[f]-\int_{x_{n n}}^{b} f(x) \omega(x) d x \leqslant I_{n}[f] .
\end{aligned}
$$

It remains to show that $I_{n}[f] \leqslant I[f]$. When $s=1$, this follows from Lemma 3.2(i) with $k=n+1$ since $I_{n}[f]=\sum_{j=1-r}^{n} \lambda_{n j} f\left(x_{n j}\right)$. When $s=0$, we shall prove that $I_{n}[f] \leqslant I[f]$ using the ideas in Freud's proof of Lemma III.1.5 in [1], which appears to be faulty.

Let $\varepsilon>0$ be arbitrary and define $f_{\varepsilon}=f(x)+\varepsilon x^{2 n+r}$. Let $H(x)$ be the polynomial of degree $2 n+r-1$ satisfying

$$
\begin{array}{ll}
H\left(x_{n j}\right)=f_{\varepsilon}\left(x_{n j}\right), & j=1-r(1) n, \\
H^{\prime}\left(x_{n j}\right)=f_{\varepsilon}^{\prime}\left(x_{n j}\right), & j=1(1) n .
\end{array}
$$


As is well known, $I_{n}\left[f_{\varepsilon}\right]=I_{n}[H]=I[H]$. Hence, it suffices to show that $G(x) \geqslant 0$ in $(a, b)$, where $G(x)=f_{\varepsilon}(x)-H(x)$, since this implies that $I_{n}\left[f_{\varepsilon}\right] \leqslant I\left[f_{\varepsilon}\right]$, and by letting $\varepsilon$ tend to zero, we get that $I_{n}[f] \leqslant I[f]$. Now, since $f_{\varepsilon}(x) \rightarrow \infty$ as $x \rightarrow b$, $G(x)>0$ in a neighborhood of $b$. Furthermore, $G(x)$ has $n$ double zeros in $(a, b)$, and when $r=1$, it has a simple zero at $x=a$ for a total of $2 n+r$ zeros. Now, if $G\left(x_{0}\right)<0$ for some $x_{0} \in(a, b)$, then $G(x)$ would have a zero of odd multiplicity at some point in $(a, b)$ and the total number of zeros would be at least $2 n+r+1$. By Rolle's theorem, there would exist a point $\xi \in(a, b)$ such that $G^{(2 n+r)}(\xi)=0$. But $H^{(2 n+r)}(x) \equiv 0$ and $f_{\varepsilon}^{(2 n+r)}(\xi)=f^{(2 n+r)}(\xi)+\varepsilon(2 n+r) !>0$, since $f^{(2 n+r)}(x) \geqslant 0$ in $(a, b)$. This contradiction proves our result.

(b) is similar.

Unfortunately, the behavior of $\lambda_{n n}, b-x_{n n}, x_{n n}-x_{n, n-1}$, and so on, have not been thoroughly investigated for general weights and there seems to be no analogue of Lemma 3.4. Thus we are not able to prove results as general as those in Sections 4 and 5 , but can prove results for the generalized Jacobi weight functions. These weight functions were studied by Nevai and others and are defined as follows:

Definition 6.2 [3]. $\omega$ is a generalized Jacobi weight function $(\omega \in G J)$ if $\omega$ can be written in the form

$$
\omega(x)=\psi(x)(1-x)^{\alpha} \prod_{k=1}^{m}\left|t_{k}-x\right|^{\gamma_{k}}(1+x)^{\beta}
$$

for $-1 \leqslant x \leqslant 1$, where $-1<t_{m}<t_{m-1}<\cdots<t_{1}<1, m \geqslant 0, \alpha, \beta, \gamma_{k}>-1$, $k=1, \ldots, m$ and $\psi \geqslant 0$ satisfies $\psi^{ \pm 1} \in L^{\infty}[-1,1]$.

Note that if $\omega \in G J$, then for any $y \in(-1,1)$ such that $y \neq t_{k}, k=1, \ldots, m$, $\omega \in I B(y)$. Hence, the results of Sections 4 and 5, in particular Corollaries 4.3 and 4.4 and Theorems 5.1(i) and 5.2, hold for generalized Jacobi weight functions.

The two basic properties of $G J$ weights that we need are given in [3, p. 673]. Taking into account that if $\omega \in G J$, then $\bar{\omega} \in G J$, these properties are

$$
\begin{gathered}
1-c_{1} / n^{2}<x_{n n}<1-c_{2} / n^{2}, \\
c_{3}\left(1-x_{n n}\right)^{\alpha+1 / 2+s} / n<\bar{\lambda}_{n n}<c_{4}\left(1-x_{n n}\right)^{\alpha+1 / 2+s} / n,
\end{gathered}
$$

where, as before, $x_{n n}$ is the $n$th root of the polynomial of degree $n$ orthogonal with respect to $\bar{\omega}(x)$, and $\bar{\lambda}_{n n}$ is the Christoffel number corresponding to $x_{n n}$. The positive constants $c_{1}, c_{2}, c_{3}$ and $c_{4}$ are independent of $n$.

Theorem 6.3. Let $(a, b)=(-1,1)$ and let $\omega(x)$ be a generalized Jacobi weight function as given by (6.3). Then

(a) $E_{n}\left[(1-x)^{-\delta}\right]=O\left(n^{-2 \alpha-2+2 \delta}\right)$ if $\alpha-\delta>-1$ and $\delta>0$. Further, there exists a positive $\eta$ such that

$$
E_{n}\left[(1-x)^{-\delta}\right] \sim n^{-2 \alpha-2+2 \delta} \quad \text { whenever } \delta \in(1+\alpha-\eta, 1+\alpha) .
$$

(b) $E_{n}[-\log (1-x)]=O\left(n^{-2 \alpha-2} \log n\right)$.

Proof. (a) When $m \geqslant 1$ in (6.3) we choose $n$ sufficiently large so that $x_{n n}>t_{1}$. Then, by (6.1) and (6.4),

$$
0 \leqslant E_{n}\left[(1-x)^{-\delta}\right] \leqslant c(1+\alpha-\delta)^{-1}\left(1-x_{n n}\right)^{1+\alpha-\delta}=O\left(n^{-2 \alpha-2+2 \delta}\right)
$$

for some positive constant $c$. 
Further, by (6.5),

$$
\lambda_{n n} f\left(x_{n n}\right)=\frac{\bar{\lambda}_{n n}\left(1-x_{n n}\right)^{-\delta}}{\left(1+x_{n n}\right)^{r}\left(1-x_{n n}\right)^{s}} \geqslant \frac{c_{3}}{2 n}\left(1-x_{n n}\right)^{\alpha+1 / 2-\delta} .
$$

Hence, by (6.1),

$$
\begin{aligned}
E_{n}\left[(1-x)^{-\delta}\right] & \geqslant \int_{x_{n n}}^{1} \omega(x)(1-x)^{-\delta} d x-\frac{c_{3}}{2 n}\left(1-x_{n n}\right)^{\alpha+1 / 2-\delta} \\
& \geqslant \hat{c} \frac{\left(1-x_{n n}\right)^{-\delta+\alpha+1}}{(1+\alpha-\delta)}-\frac{c_{3}}{2 n}\left(1-x_{n n}\right)^{\alpha+1 / 2-\delta} \\
& =\left(1-x_{n n}\right)^{-\delta+\alpha+1}\left[\frac{\hat{c}}{1+\alpha-\delta}-\frac{c_{3}}{2 n}\left(1-x_{n n}\right)^{-1 / 2}\right] \\
& \geqslant\left(1-x_{n n}\right)^{-\delta+\alpha+1}\left[\frac{\hat{c}}{1+\alpha-\delta}-\frac{c_{3}}{2} \cdot c_{1}^{-1 / 2}\right] .
\end{aligned}
$$

Hence

$$
\exists \eta>0 \ni E_{n}\left[(1-x)^{-\delta}\right] \geqslant c\left(1-x_{n n}\right)^{\alpha+1-\delta}
$$

whenever $\delta \in(1+\alpha-\eta, 1+\alpha)$; i.e.,

$$
E_{n}\left[(1-x)^{-\delta}\right] \sim n^{-2 \alpha-2+2 \delta} .
$$

(b) is similar to the first part of (a).

For Jacobi weights, we obtain the following more precise result.

THEOREM 6.4. Let $(a, b)=(-1,1)$, and let $\omega(x)$ be the Jacobi weight function $\omega^{(\alpha, \beta)}(x), \alpha, \beta>-1$. Let $J_{\nu}(x)$ be the Bessel function of the first kind of order $\nu$ and $j_{1 \nu}$ its first positive zero, where $\nu=\alpha+s$.

(a) Let $0<\delta<1+\alpha$ and

$$
s_{n}=2^{-\beta}\left(j_{1 v}^{2} / 2\right)^{-1-\nu+\delta} n^{2 \alpha+2-2 \delta}(1+\alpha-\delta) E_{n}\left[(1-x)^{-\delta}\right] .
$$

Then

$$
\max \left\{0,1-c_{0}^{2}(\nu)(1+\alpha-\delta)\right\} \leqslant \liminf _{n \rightarrow \infty} s_{n} \leqslant \limsup _{n \rightarrow \infty} s_{n} \leqslant 1
$$

where

$$
c_{0}(\nu)=2 /\left(j_{1 \nu} J_{\nu}^{\prime}\left(j_{1 \nu}\right)\right)
$$

(b) Let

$$
t_{n}=2^{-\beta-1}\left(j_{1 v}^{2} / 2\right)^{-1-\nu} n^{2 \alpha+2}(\log n)^{-1}(1+\alpha) E_{n}[-\log (1-x)] .
$$

Then

$$
\max \left\{0,1-c_{0}^{2}(\nu)(1+\alpha)\right\} \leqslant \liminf _{n \rightarrow \infty} t_{n} \leqslant \limsup _{n \rightarrow \infty} t_{n} \leqslant 1 .
$$

Proof. For the Jacobi weight function we have that

$$
\bar{\omega}(x)=(1-x)^{\nu}(1+x)^{\beta+r} .
$$


Now, by Theorem 8.1.2 in Szegö [6] (cf. [2, p. 233]),

$$
\lim _{n \rightarrow \infty} n^{2}\left(1-x_{n n}\right)=j_{1 v}^{2} / 2
$$

Hence,

$$
\begin{aligned}
\int_{x_{n n}}^{1}(1-x)^{-\delta} \omega(x) d x & \cong 2^{\beta}\left(1-x_{n n}\right)^{1+\alpha-\delta} /(1+\alpha-\delta) \\
& \cong 2^{\beta}\left(j_{1 \nu}^{2} /\left(2 n^{2}\right)\right)^{1+\alpha-\delta} /(1+\alpha-\delta)
\end{aligned}
$$

by (6.9). Further, by (15.3.11) in Szegö [6, p. 350],

$$
\bar{\lambda}_{n n} \cong 2^{\nu+\beta+r+1}\left(j_{1 \nu} / 2\right)^{2 \nu}\left\{J_{r}^{\prime}\left(j_{1 \nu}\right)\right\}^{-2} n^{-2 \nu-2},
$$

so that

$$
\begin{aligned}
\lambda_{n n}\left(1-x_{n n}\right)^{-\delta} & =\bar{\lambda}_{n n}\left(1-x_{n n}\right)^{-\delta-s}\left(1+x_{n n}\right)^{-r} \\
& \cong 2^{\nu+\beta+1}\left(j_{1 v} / 2\right)^{2 \nu}\left\{J_{\nu}^{\prime}\left(j_{1 v}\right)\right\}^{-2} n^{-2 \nu-2}\left(j_{1 \nu}^{2} /\left(2 n^{2}\right)\right)^{-\delta-s} .
\end{aligned}
$$

Then (6.6) follows easily from (6.1), (6.10), (6.11), and (6.7).

(b) is similar.

7. Interior Singularities for More General Functions. We now extend the results of Sections 4 and 5 to the function $f(x)=\phi(x) g(x)$, where $g(x)$ is smooth and $\phi(x)=|x-y|^{-\delta}$ or $\phi(x)=-\log |x-y|$. Throughout, without further mention, we assume $y \in(a, b)$.

We state first our result on avoiding the singularity.

Theorem 7.1. Assume $\omega(x) \in I B(y)$. Assume $g \in C[a, b]$.

(i) Let $f(x)=|x-y|^{-\delta} g(x), x \in[a, b] \backslash\{y\}$, where $0<\delta<1$.

(a) If $g \in \operatorname{Lip}(1-\delta)$ in $[a, b]$ and $g \in \operatorname{Lip}(1)$ near $y$, then

$$
E_{n}^{* *}[f]=O\left(n^{-1+\delta}\right), \quad E_{n}^{*}[f]=O\left(n^{-1+\delta}\right) .
$$

(b) If, further, there exists $0<\varepsilon<\delta$ such that $g \in \operatorname{Lip}(1-\varepsilon)$ in $[a, b]$ and $g^{\prime} \in \operatorname{Lip}(\delta-\varepsilon)$ near $y$, and if $g(y) \neq 0$, then

$$
E_{n}^{* *}[f] \sim g(y) n^{-1+\delta} .
$$

Further, $E_{n}^{*}[f] \sim g(y) n^{-1+\delta}$ if $\delta$ is close enough to 1 .

(ii) Let $f(x)=(-\log |x-y|) g(x), x \in[a, b] \backslash\{y\}$.

(a) If $g \in \operatorname{Lip}(1 ;-1)$ in $[a, b]$ and $g \in \operatorname{Lip}(1)$ near $y$, then

$$
E_{n}^{* *}[f]=O\left(n^{-1} \log n\right), \quad E_{n}^{*}[f]=O\left(n^{-1} \log n\right) .
$$

(b) If, further, there exists $0<\eta<1$ such that $g \in \operatorname{Lip}(1 ;-1+\eta)$ in $[a, b]$ and $g^{\prime} \in \operatorname{Lip}(0 ; \eta)$ near $y$, and if $g(y) \neq 0$, then

$$
E_{n}^{* *}[f] \sim g(y) n^{-1} \log n .
$$

Proof. See the proof of Theorem 7.5 in [2].

The following result analyzes the error when the singularity is ignored. 
THEOREM 7.2. (i) Assume $\omega(x) \in I B(y)$ for each $y$ interior to $(a, b)$. Then, given $\varepsilon>1$, there is a set $\mathscr{E}_{\varepsilon}$ in $(a, b)$ of linear Lebesgue measure zero with the following property: If $g \in \operatorname{Lip}(1)$ in $[a, b]$, then

$$
E_{n}\left[|x-y|^{-\delta} g\right]=O\left(n^{-1+2 \delta}(\log n)^{\delta}(\log \log n)^{\varepsilon \delta}\right)
$$

for all $0<\delta<1$, whenever $y \notin \mathscr{E}_{\varepsilon}$.

Hence, if $\delta<\frac{1}{2}, E_{n}\left[|x-y|^{-\delta} g\right] \rightarrow 0$ as $n \rightarrow \infty$ for almost all $y \in(a, b)$.

(ii) Assume $(a, b)=(-1,1)$, and $\omega(x)$ is the Jacobi weight function $\omega^{(\alpha, \beta)}(x)$, where $\alpha+s, \beta+r= \pm \frac{1}{2}$. Then there is a set $\mathscr{E}$ in $(-1,1)$ of linear Lebesgue measure zero with the following property: If $g \in \operatorname{Lip}(1)$ in $[a, b]$, then

$$
\left|E_{n}\left[|x-y|^{-\delta} g\right]\right| \geqslant c|g(y)| n^{-1+2 \delta}(\log n)^{\delta}(\log \log n)^{\delta}
$$

for infinitely many integers $n$ and all $0<\delta<1$, whenever $y \notin \mathscr{E}$. Here $c$ is a positive constant independent of $g, n, y$, and $\delta$.

Thus, provided the set of zeros of $g$ has linear Lebesgue measure zero, and if $\delta \geqslant \frac{1}{2}$, $E_{n}\left[|x-y|^{-\delta} g\right] \nrightarrow 0$ as $n \rightarrow \infty$ for almost all $y \in[a, b]$.

(iii) Assume $(a, b)=(-1,1)$ and that $\omega(x)$ is the Jacobi weight function $\omega^{(\alpha, \beta)}(x)$, where $\alpha$ and $\beta$ satisfy (4.8). Then, if $y=\cos \pi \xi$, where $\xi$ is an irrational number, and $g \in \operatorname{Lip}(1)$ in $[-1,1]$, then

$$
\left|E_{n}\left[|x-y|^{-\delta} g\right]\right| \geqslant c|g(y)| n^{-1+2 \delta}
$$

for infinitely many $n$ and all $0<\delta<1$. Here, $c$ is a positive constant independent of $g$, $n$, and $\delta$. Hence, for any $y \ni g(y) \neq 0$, if $\delta \geqslant \frac{1}{2}, E_{n}\left[|x-y|^{-\delta} g\right] \nrightarrow 0$ as $n \rightarrow \infty$.

Proof. See the proof of Theorem 7.6 in [2].

In a similar fashion, one can use Theorem 5.2 to prove the following result for ignoring a logarithmic singularity:

TheOREM 7.3. Assume $\omega(x) \in I B(y)$ for each $y$ interior to $(a, b)$. Then there is a set $\mathscr{E}$ of linear Lebesgue measure zero (even further of Hausdorff dimension zero) with the following property: If $g \in \operatorname{Lip}(1)$ in $[a, b]$, then

$$
E_{n}[(-\log |x-y|) g]=O\left(n^{-1} \log n\right), \quad \text { whenever } y \notin \mathscr{E} .
$$

8. Endpoint Singularities for More General Functions. In extending the results of Section 6 to more general functions, we shall assume throughout that $(a, b)=(-1,1)$ and that $\omega(x)$ is a generalized Jacobi weight function given by (6.3). Our main result for endpoint singularities is as follows:

THEOREM 8.1. (i) Let $0<\delta<\min \{1,1+\alpha\}$, and let $l$ be the smallest integer $\geqslant 2(1+\alpha-\delta)$. Let $g \in C^{\prime}[-1,1]$ and assume there exists $\eta>0$ such that $g^{(l)}(x)$ $\in \operatorname{Lip}(\delta ; \eta)$ near 1 . Then

$$
E_{n}\left[(1-x)^{-\delta} g\right]=O\left(n^{-2(1+\alpha-\delta)}\right) .
$$


(ii) Let $k$ be the smallest integer $\geqslant 2(1+\alpha)$. Let $g \in C^{k}[-1,1]$ and assume there exists $\eta>1$ such that $g^{(k)}(x) \in \operatorname{Lip}(0 ; \eta)$ near 1 . Then

$$
E_{n}[(\log (1-x)) g]=O\left(n^{-2(1+\alpha)} \log n\right) .
$$

Proof. See the proof of Theorem 8.2 in [2] with $\nu$ replaced by $\alpha$.

Department of Applied Mathematics

The Weizmann Institute of Science

Rehovot 76100, Israel

1. G. Freud, Orthogonal Polynomials, Pergamon Press, New York, 1966.

2. D. S. LUBINSKY \& P. RABINOWITZ, "Rates of convergence of Gaussian quadrature for singular integrands," Math. Comp., v. 43, 1984, pp. 219-242.

3. P. NeVAI, “Mean convergence of Lagrange interpolation. III," Trans. Amer. Math. Soc., v. 282, 1984 , pp. 669-698.

4. P. Rabinowitz, "Ignoring the singularity in numerical integration," in Topics in Numerical Analysis III (J. J. H. Miller, ed.), Academic Press, London, 1977, pp. 361-368.

5. P. Rabinowitz, "Numerical integration in the presence of an interior singularity," J. Comput. Appl. Math. (To appear.)

6. G. Szegö, Orthogonal Polynomials, rev. ed., Amer. Math. Soc. Colloq. Publ., vol. 23, Amer. Math. Soc., Providence, R. I., 1959. 